\title{
Stiff-Person Syndrome
}

National Institute of Neurological Disorders and Stroke (NINDS)

\section{Source}

National Institute of Neurological Disorders and Stroke (NINDS). Stiff-Person Syndrome Information Page.

Stiff-person syndrome (SPS) is a rare neurological disorder with features of an autoimmune disease. SPS is characterized by fluctuating muscle rigidity in the trunk and limbs and a heightened sensitivity to stimuli such as noise, touch, and emotional distress, which can set off muscle spasms. Abnormal postures, often hunched over and stiffened, are characteristic of the disorder. People with SPS can be too disabled to walk or move, or they are afraid to leave the house because street noises, such as the sound of a horn, can trigger spasms and falls. SPS affects twice as many women as men. It is frequently associated with other autoimmune diseases such as diabetes, thyroiditis, vitiligo, and pernicious anemia. Scientists don't yet understand what causes SPS, but research indicates that it is the result of an autoimmune response gone awry in the brain and spinal cord. The disorder is often misdiagnosed as Parkinson's disease, multiple sclerosis, fibromyalgia, psychosomatic illness, or anxiety and phobia. A definitive diagnosis can be made with a blood test that measures the level of glutamic acid decarboxylase (GAD) antibodies in the blood. People with SPS have elevated levels of GAD, an antibody that works against an enzyme involved in the synthesis of an important neurotransmitter in the brain. 